\title{
UNA INYECCIÓN SOCIOLÓGICA AL MODELO DE ACEPTACIÓN DE LA TECNOLOGÍA \\ Respuesta a nuestros críticos
}

\section{A SOCIOLOGICAL INJECTION TO THE TECHNOLOGY ACCEPTANCE MODEL}

Reply to our critics

Cristóbal Torres Albero cristobal.torres@uam.es

Universidad Autónoma de Madrid. España

José Manuel Robles Morales jmrobles@ccee.ucm.es

Universidad Complutense de Madrid. España

Óscar Molina Molina omolina@iesa.csic.es

Instituto de Estudios Sociales de Andalucía. España

Consejo Superior de Investigaciones Científicas

INTRODUCCIÓN

La critica formulada a nuestro artículo "¿Por qué usamos las tecnologías de la información y las comunicaciones? Un estudio sobre las bases sociales de la utilidad individual de Internet" publicado en la Revista Internacional de Sociología nos ha deparado un doble motivo de satisfacción.

En primer lugar porque, aunque ya hace mucho tiempo de aquel célebre diagnóstico de las ciencias sociales en España que afirmaba que "la mejor forma de guardar un secreto en nuestro país era publicarlo.... puesto que nadie se molestaba en leer lo que los colegas publicaban", no es habitual que, de manera espontánea, la comunidad académica de las ciencias sociales realice réplicas y críticas a investigaciones concretas. De la misma manera, tampoco es habitual que las revistas dediquen su escaso y cotizado espacio a este tipo de ejercicios. Pero aunque no sea algo usual, sí existe una creciente implicación en los procesos de validación comunal del conocimiento científico. Y el texto de nuestros críticos no solo es una muestra de este cambio de pauta sino que, además, es un ejemplo de sensatez, crítica constructiva y propuesta de integración 
interdisciplinar. Y este hecho es el segundo motivo de nuestra satisfacción. Con independencia de los argumentos que formulamos más adelante, y que en buena medida tienen que ver con el hecho de que el campo de especialización de nuestros autores es ajeno a la tradición sociológica a la que vinculamos la investigación referida, el texto que ahora nos ocupa nos ha proporcionado valiosa información tanto para complementar nuestra aproximación teórica, como para mejorar en futuras investigaciones el eje metodológico en el que los autores incluyen los tres sesgos (estructural, instrumental y metodológico) que nos atribuyen. Sin embargo, no nos gustaría dejar pasar la oportunidad de aclarar algunas cuestiones relativas a sus comentarios.

\section{DISCUSIÓN DE LA REVISIÓN CRÍTICA}

Nuestra investigación teórica y empírica sobre el modelo TAM (Technological Acceptance Model) parte de la necesidad de integrar en los debates e investigaciones de la sociología de la ciencia y la tecnología aquellas propuestas que, ajenas al origen y desarrollo de esta especialidad, han mostrado una gran fertilidad académica. El modelo TAM surge en el ámbito de la psicología para aplicarse, desde un prisma individualista metodológico, a campos cada vez más diversos. Compartimos la propuesta integradora de las ciencias sociales, así como el carácter complementario de las ciencias del comportamiento humano, que plantean algunos especialistas en la teoría sociológica analítica. Por esta razón, la aplicación a nuestros estudios del modelo TAM era, y sigue siendo, una necesidad percibida y asumida por nosotros, dando por supuesto que nuestra preocupación intelectual está dirigida hacia los problemas sustantivos (dimensión interna) de la sociología de la ciencia y la tecnología, y que nuestra filiación institucional (dimensión externa) se encuentra en la más general disciplina de la sociología. Partiendo de estas premisas, nuestra estrategia en la elaboración del artículo fue: (a) presentar los rasgos básicos del modelo TAM en el ámbito de la sociología española, asumiendo su valía explicativa en sus campos de desarrollo y (b) complementar la base que supone el TAM con un enfoque sociológico. Una vez presentados nuestros objetivos, pasamos a discutir los comentarios presentados más arriba.

Nuestros críticos señalan que la evolución del TAM ha supuesto un cambio de modelo (TAM2) que excluye las actitudes e incorpora otros factores determinantes como los procesos de influencia social y los factores relativos a los procesos cognitivos. Sin duda, dada la compleja evolución histórica del modelo TAM (recogida en nuestro artículo) será posible encontrar alguna insuficiencia en los epígrafes dedicados al estado de la cuestión y al giro sociológico, máxime cuando el repaso del estado de la cuestión no era sino una base (limitada en espacio) sobre la que plantear nuestra investigación empírica y orientar nuestra conclusión a favor del giro sociológico. Pero estimamos que tal afirmación de exclusión de las actitudes no se corresponde con la amplia diversidad de matices y orientaciones que se dan entre los seguidores del TAM2. El propio estudio 
que exponemos de Venkatesh et al. (2003), al que también recurren nuestros colegas para formular su argumento, así lo atestigua.

En cualquier caso, tomamos muy en consideración las actitudes descritas por la TAM debido a que, siguiendo a Elster (La explicación del comportamiento social: más tuercas y tornillos para las ciencias sociales, 2010), consideramos este tipo de motivaciones sociales como uno de los elementos más importantes en la explicación sociológica del comportamiento. El hecho de incluir las variables sociodemográficas en el modelo TAM es precisamente un intento de mejorar nuestra comprensión de las actitudes bajo la premisa de la influencia de dicho tipo de variables sobre las actitudes individuales. Creemos que, para una buena explicación sociológica, no es suficiente, como es práctica frecuente, con afirmar que una acción determinada (usar Internet) es racional, útil o funcional. Es decir, no es suficiente con referirse a las consecuencias. Es necesario comprender si las intenciones del agente estaban o no en concordancia con la acción realizada, así como con las consecuencias de la misma. Las intenciones de uso conforman la dimensión más propia de la psicología, pero el uso de Internet (la acción) y, sobre todo, la relación entre intenciones, acción y las consecuencias de la acción es el ámbito explicativo de lo que nosotros consideramos el ámbito más fértil de la sociología.

Por esta razón, desde un punto de vista sociológico, el efecto de la estructura social sobre las actitudes individuales no puede reducirse al efecto de variables psicosociales, tal y como plantean nuestros críticos, como los "procesos de influencia social (normas subjetivas, voluntariedad, imagen y experiencia) o cognitivos (relevancia del trabajo, calidad del resultado y demostrabilidad del resultado)".

Respecto de los sesgos en la explicación que nuestros críticos identifican debe tenerse en cuenta lo siguiente. En lo referente a lo etiquetado como sesgo estructural entendemos que el ámbito del TAM que interesa a la sociología no son, exclusivamente, las intenciones de uso sino el uso declarado de Internet. La acción (usar o uno usar Internet) es el elemento axial de la explicación. Y ello es así porque mientras que las intenciones de uso conforman una dimensión más propia de la psicología, el uso de Internet es un elemento clave en todo análisis social, aparte de una evidencia empírica fáctica. Por ello, una estrategia que pasa por orientar hacia la sociología el modelo TAM debe privilegiar las situaciones sociales creadas (como decimos, uso o no uso) a la par que situar en un segundo plano, al menos en un primer momento, el análisis de un problema (las intenciones) más proclive a la interpretación psicológica.

En relación con los sesgos instrumentales nos parece enriquecedora la propuesta de las ventajas del modelo de ecuaciones estructurales. No obstante, ese es un enfoque de análisis para un modelo TAM donde ya estén plenamente integradas tanto la aproximación psicosocial como la estructural. Pero nuestra operación era previa. Es decir, de lo que se trataba era de poner de manifiesto la pertinencia y prioridad lógica de las variables estructurales sobre las psicológicas de la percepción de la utilidad y facilidad de uso. Y corroborarlo empíricamente. Y en esa línea, la conveniencia y superioridad del análisis logístico como técnica estadística de análisis es incontestable. 
Finalmente, en relación con el sesgo metodológico también debe destacarse el valor de la crítica recibida. Sin duda, las dos propuestas referidas a la utilización de varios indicadores y de profundizar en la operacionalización del criterio de usuario de Internet (por cierto, algo que contrasta con la crítica anterior de atender no al uso sino a las intenciones de uso) son muy convenientes y debemos incorporarlas en futuras investigaciones. De nuevo aquí nuestra explicación tiene que ver con la distinta tradición de recogida de información en el campo sociológico respecto de campos como la psicología o los estudios de management organizativo donde se ha desarrollado el TAM. No obstante lo cual, como se ha indicado, asumimos dichas críticas y, en la medida de nuestras posibilidades, trataremos de incorporarlas a nuestras futuras investigaciones.

\section{Conclusiones}

En nuestra opinión el sesgo básico en el que incurren nuestros críticos se explicita tanto en su epígrafe introductorio como en las conclusiones que formulan. En esos párrafos se registra su desacuerdo con la idea, que nos atribuyen, de la "preponderancia, superioridad o supremacía" (palabras de nuestros críticos) de las variables sociológicas frente a las psicológicas. Aunque es cierto que en un único pasaje usamos la expresión de "preponderancia de las variables sociales sobre las psicológicas", nuestras constantes afirmaciones se limitan a constatar la mayor capacidad explicativa de variables como la edad o la educación sobre la utilidad o la facilidad de uso percibida y, con ello, la necesidad de integrarlas en el TAM y de proceder a un giro sociológico. Y en las conclusiones, explícitamente, renunciamos a volver a los tiempos históricos de respectivos reduccionismos excluyentes entre la sociología y la psicología. La constatación empírica se debe, en buena medida, a que nuestra evidencia empírica proviene de una muestra representativa de la población española y no de un grupo social concreto con rasgos sociodemográficos muy homogéneos. Algo habitual en los estudios empíricos del modelo TAM.

Pero más allá de la distinta filiación institucional y de tradiciones teóricas entre ambos grupos de autores que expresa esta diferente interpretación de un mismo texto, nuestros críticos terminan su nota en la misma línea argumental con la que nosotros iniciamos nuestras conclusiones. Es decir, no se trata de concebir ambas perspectivas como excluyentes (algo ya superado en la teoría social) sino de integrarlas en un modelo socio-psicológico. Para lo que era necesaria nuestra operación del giro sociológico del TAM, dado que sus campos de desarrollo han sido los ya referidos de la psicología y los estudios de management de las organizaciones, y el tratamiento que estos campos dan a las variables sociales no coincide con aquellas que la tradición sociológica ha identificado como más relevantes. En definitiva, de nuestro estudio no creemos que pueda construirse, como apuntan nuestros críticos, un modelo estrictamente sociológico del TAM sino el socio-psicológico por el que ellos mismos también apuestan. 
No queremos dejar de terminar la contestación a la réplica recibida sin expresar a nuestros críticos nuestro más sincero agradecimiento por la desinteresada tarea asumida. No solo porque va a enriquecer nuestro futuro trabajo de investigación y discusión teórica, sino porque estimamos que también contribuye al progreso del campo ocupado en la indagación sobre el uso de las TIC. Una explicación en la filosofía y sociología de la ciencia respecto de la baja frecuencia de debates de este tipo en las comunidades científicas argumenta que en la república de la ciencia se evita cualquier polémica sobre una investigación (aunque se esté en desacuerdo) cuando se trata de aspectos que el resto de investigadores consideran irrelevantes. Es una mera cuestión de optimización de energías y recursos siempre escasos. Con su aportación, nuestros críticos coinciden con nosotros en la importancia de desarrollar el señalado modelo socio-psicológico y apuntan otros argumentos que enriquecen esta misma línea.

Pero más allá del beneficio que extraemos de su crítica y del progreso intelectual que estimamos supone para el referido campo, el trabajo asumido por nuestros críticos también expresa su compromiso con el ethos de valores morales de construcción y validación comunitaria del conocimiento científico.

CRISTÓBAL TORRES ALBERO es Catedrático de universidad en el Departamento de Sociología de la Universidad Autónoma de Madrid. Su campo de trabajo prioritario es la sociología de la ciencia y la tecnología así como los estudios sociales de la sociedad de la información y del conocimiento.

JOSÉ MANUEL ROBLES MORALES es Profesor Titular (interino) de la Sección departamental de Sociología III en la Facultad de Ciencias Económicas y Empresariales de la Universidad Complutense de Madrid. Está interesado en la relación entre tecnología, sociedad y política y es especialista en participación política, acción colectiva, democracia digital y brecha digital.

ÓSCAR MOLINA MOLINA es Experto Universitario en Estadística Pública y en Métodos Avanzados de Estadística Aplicada. Ha trabajado durante dos años en la Unidad Estadística de la Consejería de Cultura, donde ha colaborado en varios proyectos. Desde 2005 trabaja en el Instituto de Estudios Sociales Avanzados (IESA-CSIC), donde realiza labores de apoyo estadístico a diversos proyectos, dedicándose principalmente a las líneas de investigación sobre nuevas tecnologías, turismo e inmigración. 